\title{
Infant Monitoring and Fall Avoidance System using Tri-Axial Accelerometer and ARM7 Microcontroller
}

\author{
Aryan Kaushik \\ Student \\ EECE Department, \\ ITM University, Gurgaon
}

\author{
Ramandeep Singh \\ Assistant Professor \\ EECE Department, \\ ITM University, Gurgaon
}

\begin{abstract}
This paper describes system for monitoring and fall controlling of infants using tri-axial accelerometer together with ARM7 microcontroller used as a processing system to prevent fall of infants. The system is composed of data acquisition, fall controlling and monitoring and processing unit for analysis. Tri-axial accelerometer is used for human position tracking and fall detection. The system is capable of monitoring infants in real time and the obtained calibrated results are taken into consideration. The system including operation of accelerometer and the processing unit is explained in detail.
\end{abstract}

\section{General Terms}

ARM7 micro-controller, tri-axial accelerometer, ADXL335, LCD, GPS.

\section{Keywords}

Monitor and fall control, data acquisition, DC (Duty Cycle), RS (Region Select), B (Buzzer bit).

\section{INTRODUCTION}

Falls of infants are one of the leading health problems for them. They can occur in home and anywhere else. Falls increase risk for serious injuries, long term disability and loss of independence. A fear of fall of an infant does not allow the caretaker to go away from the infant and to perform his/her other concerns. Fall of infants from certain height is considered as one of the major health concerns by the pathologists. Presently in medical services, prevention is preferred over the treatments and health management is considered primarily instead of the disease-control efforts [1]. Detection of a fall possibly leading to injury in timely manner makes the prevention easier. Presently, there are following fall detection systems that can be considered [2][3] as follows:

- User activated alarm systems (wireless tags),

- Computer vision systems,

- Visual fall detection,

- Mobile fall detection systems,

- Wearable sensors (accelerometers, gyroscopes, contact sensors and switches),

- Acoustic fall detection, which is a method to detect accidents and reporting it using acoustic's method is described in [4]

The most common method for fall detection is using a triaxial accelerometer. An easy and simple method, to detect fall of infants and monitoring and controlling it, is presented in this paper. There have been researches which discuss on distinguishing daily life activities using various sensors and discrimination algorithms [5] [6] [7]. There are many research groups studying about human activity recognition using various sensors like cameras, GPS, accelerometers. Tapia used a simple state change sensor which detects the change of state of objects used at home [8], and Han made use of an infrared camera to contrast the silhouette of the user and the obtained sequence of images helped him in recognizing user's activity [9]

In the robotics field, human gestures have been recognized, by attempting several researches, using vision based systems [10] [11], sensors capturing motion [12] [13], or using active tracking mechanism based finger gesture recognition systems [14]. The activity of the infant is a major concern in this research, which further decides the way of monitoring and controlling. The gestures of infant can be recognized by implementing the following two systems: sensor-based and vision based, [15].The sensor-based solutions use accelerometers or gyros for detecting the gestures. When gestures are done with the hands, a special glove or special device kept in hand is used. When gestures are done with the body, sensors are mounted on the part of the body used for communication. This paper presents a body gesture recognition system built around an accelerometer sensor. The whole system consists of an accelerometer which the infant wears on himself, local units to collect data that are placed in infant's vicinity and systems for collecting, processing and displaying the calibrated data. The system is divided into two units. One is the monitor and fall control unit and the other unit is data acquisition unit, which consists of the infant wearing accelerometer (ADXL335), which is small and lightweight and transmits command packets to ARM7microcontroller. The monitor and fall control unit consists of a processing unit (ARM7 microcontroller), $16 \times 2$ $L C D$, Switch keypad and Buzzer. LCD shows the calibrated output values. ARM7 microcontroller has inbuilt $A D C$, which converts the obtained analogue signal into the digital output and Buzzer provides a sound signal showing the fall detection resulting into appropriate actions to take place. A wired connection is provided between both the units, which helps in communicating between them.

The other unit describes three regions:

a) Safe Region

b) Warning Region,

c) Alert Region

The place of accelerometer is considered as a reference region which by then describes the above mentioned three regions. 


\section{INTRODUCTION TO [ADXL335] ACCELEROMETER}

An accelerometer is a device that measures proper acceleration. The proper acceleration measured by an accelerometer is not necessarily the coordinate acceleration (rate of change of velocity). Instead, the accelerometer sees the acceleration associated with the phenomenon of weight experienced by any test mass at rest in the frame of reference of the accelerometer device. Accelerometers have multiple applications in industry and science. Single-axis and multiaxis models of accelerometer are available to detect magnitude and direction of the proper acceleration (or gforce), as a vector quantity, and can be used to sense orientation (because direction of weight changes), coordinate acceleration (so long as it produces g-force or a change in gforce), vibration, shock, and falling in a resistive medium (a case where the proper acceleration changes, since it starts at zero, then increases).

An accelerometer measures proper acceleration, which is the acceleration it experiences relative to freefall and is the acceleration felt by people and objects. Put another way, at any point in space-time the equivalence principle guarantees the existence of a local inertial frame, and an accelerometer measures the acceleration relative to that frame. Accelerometers hold the basic applications in engineering, biology, industry, building and structural monitoring, medical domain, navigation, image stabilization, orientation sensing and many more. MEMS (micro electro-mechanical systems) accelerometers have been widely used in automotive but much recently, MEMS low g accelerometers started to enter consumer applications, in large volume, as free fall detection for hard disk drives and as motion detection for game control. There are many high performance accelerometers which are having a low noise floor [16][17][18], however, these accelerometers are only sensitive to single-axis. This paper makes use of ADXL335 which is mounted on a jacket, which the infant wears.

\section{EXISTING TECHNOLOGY USING ACCELEROMETER}

Based on its wide range of applications, accelerometer is highly used in various existing technologies:

- To count energy expenditure over a wide range of physical activities,

- Accurate and fast fall detection,

- Intelligent bridge maintenance system using MEMS (micro electro-mechanical systems) along with the network technology,

- Objective measurement of functional upper-extremity movement,

- Real-time pothole detection using android smart-phones with accelerometers.

\section{SYSTEM ARCHITECTURE}

In this section, the operation of the whole system through its functions and to the detection of fall is described. The figures have been simplified for better understanding of the system. Block diagram of the monitor and fall control unit is mentioned in the section along with the criteria to identify the specific regions for an infant and setup of the accelerometer placed in infant's vicinity. The process of acquisition of the data is done by the data acquisition unit.

\subsection{Monitor and Fall Control}

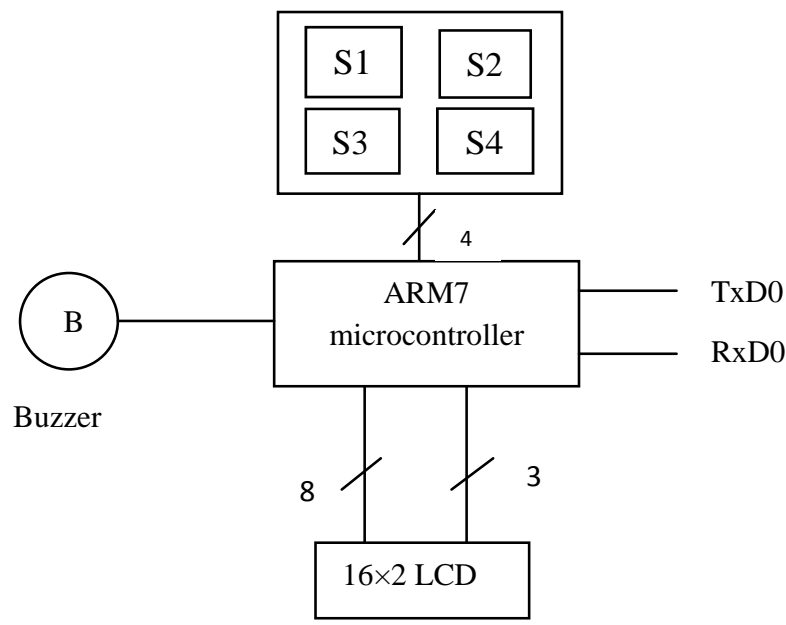

Figure 1: Block Diagram for monitor and fall control unit

As shown in figure1, the monitor and fall control unit consists of ARM7 microcontroller which connects 8 data lines and 3 control lines to $16 \times 2$ LCD. LCD shows the calibrated output values, which describes the location of the accelerometer and accordingly the place of the infant considering two dimensional view.ARM7 microcontroller has inbuilt ADC, where the analogue input is converted to the digital one, to conduct the processing by the microcontroller. Buzzer is used to produce various audio signals for the various locations of accelerometer in the predefined regions. Keypad matrix, which makes use of switches performing different functions, is used to activate or deactivate the operations performed by the accelerometer in different locations. Switch S1 activates or deactivates the operation for safe region, Switch S2 activates or deactivates the operation for waiting region, whereas Switch S3 performs the same operation for alert region. Switch $\mathrm{S} 4$ is used to reset values for all the regions. This provides a convenience to the system to be user friendly.

\subsection{DATA ACQUISTION}

The 3-axis accelerometer transmits analogue inputs to the various pins of microcontroller, where these inputs are converted into digital ones. AD0.4 is 9th pin of IC, which can be symbolised as P0.25. AD0.6 is 27 th pin of IC, which can be symbolised as P0.4 and AD0.7 is 29th pin of IC, which can be symbolised as P0.5. Vss pin is used for a $0 \mathrm{~V}$ reference whereas Vdd is the power supply voltage $(3.3 \mathrm{~V})$ for the core and $\mathrm{I} / \mathrm{O}$ ports. TxD0 pin is used as a transmitter output for UART0 and RxD0 pin is used as a receiver input for UART0 of the microcontroller.

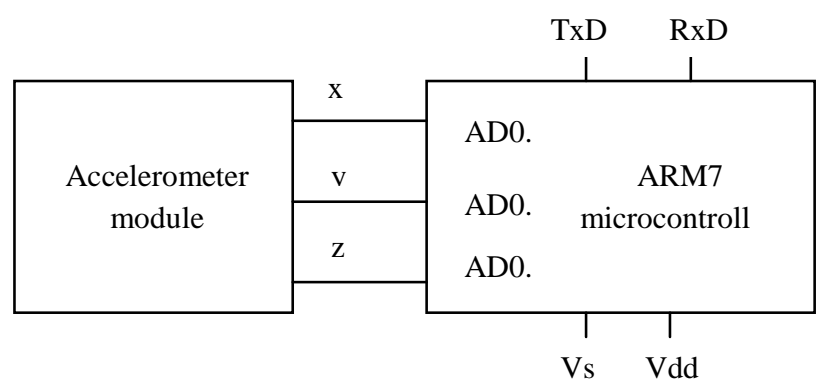

Figure 2: Connections of accelerometer module with ARM7 microcontroller pins 
The data acquisition unit consists of accelerometer module, which sends the measurement of movement of the infant in all the three directions: $\mathrm{x}, \mathrm{y}$ and $\mathrm{z}$, and ARM7 microcontroller which processes the various measurements send by the accelerometer along with the processing of the command packet. ARM7 microcontroller has an inbuilt ADC (Analogue to Digital Convertor), which converts the input signals coming from the accelerometer module to the digital ones for the required processing of the signals.

\subsection{REGION IDENTIFICATION}

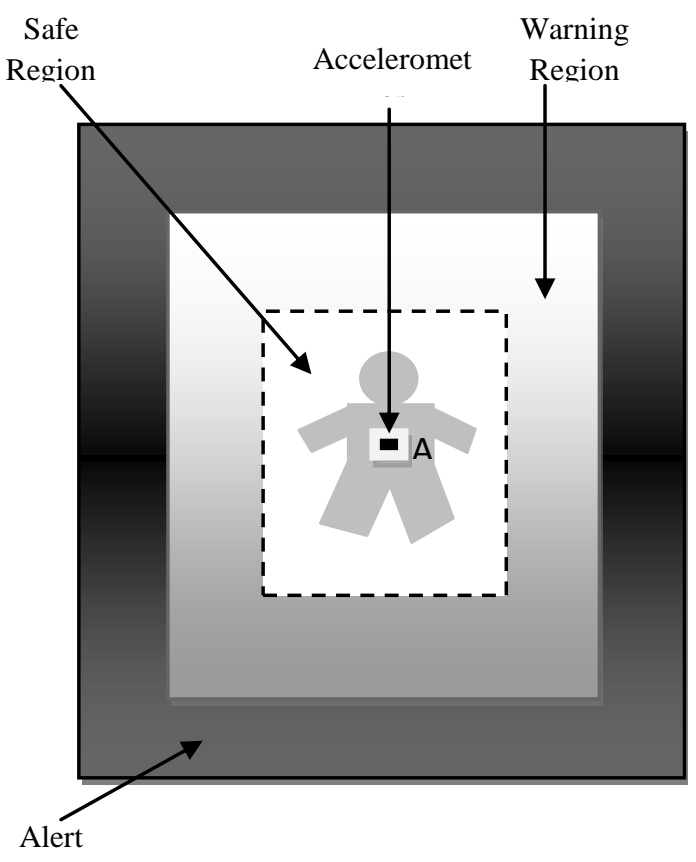

Region

\section{Figure 3: Specification of the regions in infant's vicinity}

The infant wears the jacket which consists of an accelerometer (A). It is very practical because of portability and the ability to be mounted in small pockets inside the clothes of infants. The measurement data is sent to the monitor and fall control unit, where the microcontroller processes the received signals accordingly. The topic of accelerometer based activity recognition is not new. Bao and Intille [19] identified twenty activities using bi-axial accelerometers placed in five locations on the user's body, which was represented as a activity recognition system. This research makes use of ADXL335 accelerometer, which is a small, thin, low power, complete 3 -axis $3 \mathrm{~g}$ accelerometer as shown in [20], which senses the activity of infant and accordingly selects the type of region. The regions mentioned above are described as follows:

- $\quad$ Safe region is described as a region where the infant is safe and there is no need to be worried for the falling of the infant. Buzzer does not produce any sound for this region.

- Warning region is the region where Buzzer produces a short beep sound to the user which indicates the warning provided to the user to monitor the fall.
- $\quad$ Alert region describes the region where Buzzer produces a long beep sounds to indicate the final warning to rescue the infant from falling.

The ARM7 microcontroller, present in the monitor and fall control unit, gets the command packet coming from the accelerometer module which indicates the state of infant. The movement of infant is noted in all three directions: $\mathrm{x}, \mathrm{y}$ and $\mathrm{z}$. There are pre-defined boundaries for all the three regions, as mentioned above. The user gets option of making the indication for the safe region off, through the use of keypad switches provided for activation and deactivation of the particular regions. It is to avoid the beep sounds produced by the buzzer every time, when infant shows a bit movement in safe region. Also, same way can be carried out to deactivate the sounds generated by the buzzer when infant is in warning region. If the infant comes under none of the regions, S4 switch of the keypad switches can be used to deactivate the sending and processing of command signals provided to the monitor and fall control unit. In this manner, the system can be properly used to take a prior action to prevent the falling of the infant, by looking for the various regions as mentioned above. The identification of the region makes sure that the infant is safe and if he does not come under the boundaries of safe region, it becomes the responsibility of the user to check the LCD screen and listen to the sounds of buzzer to recognize the safety of the infant. The use of keypad switches does not come under consideration, when the whole system is not being used to compute the decision of indicating the particular region in which the infant is present. The best use of accelerometer is made by sensing the movement of the infant in every direction. A tri-axial accelerometer precisely fits this requirement of sensing the movement of infant in every possible direction. Accelerometer is a lightweight sensor which can easily be fitted into the jacket of the infant and its other specific properties such as portability makes it more valid to be used. The whole system supports the use of such an accelerometer and provides better results in identifying the regions, in which the infant makes movement or he switches over from one particular region to the other one. 


\section{FLOW DIAGRAM}

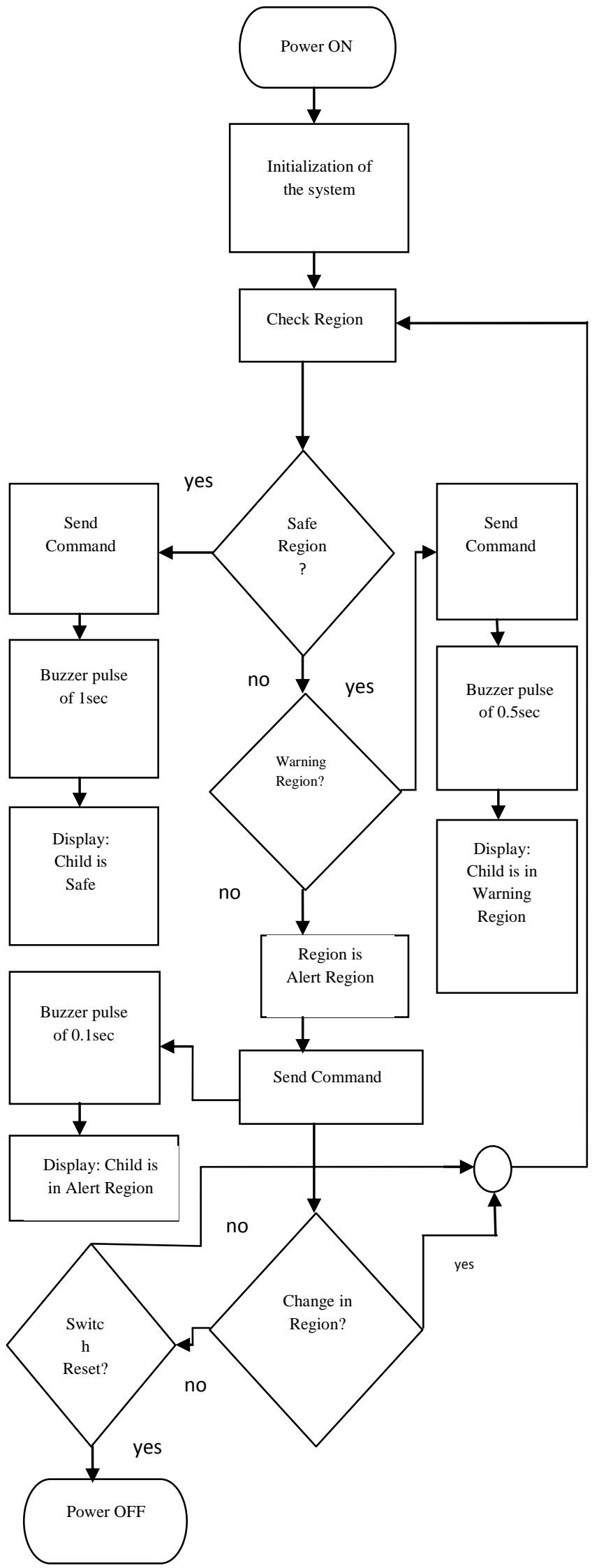

Figure 4: Algorithm for fall prevention
This algorithm is able to explain the whole mechanism of monitor and fall control unit and data acquisition unit. Initially, the system gets initialized as to proceed to note the activity of the infant and to prevent him from falling. The region, in which the infant is presently lying, gets checked. If the infant is in safe region, command packet is being sent to the monitor and fall control unit. The buzzer in this unit generates the pulse repeating after every 1 second and LCD displays a message that the child is in safe region. If the child does not fall in the safe region, it checks for the warning region. When child is in warning region, command packet, accordingly, is sent to the monitor and fall control unit and then buzzer generates a pulse repeating after every 0.5 seconds and LCD displays a message that the child is in warning region. If the child does not fall in the warning region, he is in alert region. A pulse repeating after every 0.1 seconds gets generated and LCD displays a message that the child is in alert region.

\section{COMMAND PACKET DESIGNING}

RS0 $\rightarrow$ Region Select Bit 0, RS1 $\rightarrow$ Region Select Bit 1, $\mathrm{B} \rightarrow$ Buzzer State, DC0 $\rightarrow$ Duty Cycle Bit 0 , DC1 $\rightarrow$ Duty Cycle Bit $1, X \rightarrow$ Don't Care

The accelerometer transmits a command packet of 1 byte ( 8 bits) and the microcontroller, placed in the monitor and fall control unit, receives and processes it. The packet determines

\begin{tabular}{|l|l|l|l|l|l|l|l|}
\hline $\mathbf{X}$ & $\mathbf{X}$ & $\mathbf{X}$ & DC1 & DC0 & B & RS1 & RS0 \\
\hline
\end{tabular}

the type of region in which the infant is present, the state of buzzer and the frequency of sound produced by the buzzer.

Table 1: Selection of Region by command packet

\begin{tabular}{|c|c|c|}
\hline RS1 & RS0 & Region \\
\hline 0 & 0 & Safe \\
\hline 0 & 1 & Warning \\
\hline 1 & 0 & Alert \\
\hline
\end{tabular}

Table 2: State of Buzzer determined by command packet

\begin{tabular}{|c|c|}
\hline B & State of Buzzer \\
\hline 0 & Off \\
\hline 1 & On \\
\hline
\end{tabular}

The above tables provide various indications to the user, regarding the state of infant, which he recognizes from the monitor and fall control unit. The system helps the user to prevent the infant from falling.

\section{CONCLUSION}

Tri-axial accelerometers can be used for preventing fall of infants. This paper describes the system for monitoring and fall controlling of infants using tri-axial accelerometers. The tri-axial accelerometer module, being a part of the data acquisition unit, senses the movement or activity of the infant and accordingly sends a command packet which specifies the type of region in which the infant is present, so that the infant can be prevented from falling. The monitor and fall control unit processes the incoming command packet and then indicates the type of region showing the values of dimensional movements in all the 3 axes and generating different sound pulses for each region. Time take for repetition of pulse 
generated by the buzzer also leads to the conclusion of the region, in which the infant is present.

Table 3: Time taken by the repetitive pulse generated by buzzer

\begin{tabular}{|c|c|c|}
\hline DC1 & DC0 & $\begin{array}{c}\text { Time taken for repetition } \\
\text { of pulse (sec) }\end{array}$ \\
\hline 0 & 0 & 1 \\
\hline 0 & 1 & 0.5 \\
\hline 1 & 0 & 0.1 \\
\hline
\end{tabular}

If the time taken for repetition of pulse is 1 second and both the duty cycle bits to be 0 , it indicates for safe region. Similarly, if the time taken for repetition of pulse is 0.5 seconds and duty cycle bit 1 and duty cycle bit 0 to be 0 and 1 respectively, it indicates for warning region. If the time taken for repetition of pulse is 0.1 seconds and duty cycle bit 1 and duty cycle bit 0 to be 1 and 0 respectively, it indicates for alert region.

\section{ACKNOWLEDGMENTS}

We gratefully wish to thank ITM University, in particular the Embedded Systems lab and Electronics lab, for carrying out research work

\section{REFERENCES}

[1] Park s, Jayaraman S, "Enhancing the Quality of Life Through Wearable Technology", IEEE Eng. Med. Biol. Mag., vol. 22, no 3, pp. 41-48, 2003

[2] Luštrek M.; Kaluža B.; "Fall Detection and Activity Recognition with Machine Learning"; Informatica 33 (2009) 205-212

[3] U. Lindemann; A. Hock; M. Stuber; W. Keck; C. Becker; "Evaluation of a fall detector based on accelerometers: a pilot study"; Medical \& Biological Engineering \& Computing, 2005, Vol. 43, pp. 548-551

[4] David A. Whitney and Joseph J Pisano TASC, Inc., Reading, Massachusetts, "Auto Alert: Automated Acoustic Detection of Incidents", IDEA project.

[5] C.V. Bouten,K. T.Koekkoek, M.Verduin, R.Kodde, and J. D. Janssen, "A triaxial accelerometer and portable data processing unit for the assessment of daily physical activity," IEEE Trans. Biomed. Eng., vol. 44, no. 3, pp. 136-147, Mar. 1997.

[6] M. J. Mathie, A. C. F. Coster, N. H. Lovell, and B. G. Celler, "A pilot study of long term monitoring of human movements in the home using accelerometry," $J$. Telemed. Telecare, vol. 10, pp. 144-151, 2004.

[7] K. Aminian, P. Robert, E. E. Buchser, B. Rutschmann, D. Hayoz, and M. Depairon, "Physical activity monitoring based on Accelerometry: Validation and comparison with video observation," Med. Biol. Eng. Comput., vol. 37, pp. 304-308, 1999.
[8] E. Tapia, S. Intille and K. Larson, "Activity recognition in the home using simple and ubiquitous sensors," Lecture Notes in Computer Science, Int. Conf. on Pervasive Computing, vol. 3001, pp. 158-175, 2005

[9] J. Han and B. Bhanu, "Human activity recognition in thermal infrared imagery," IEEE Computer Society Conf. on Computer Vision and Pattern Recognition, vol. 3, pp. 17-25, 2005.

[10] I. Mihara, Y. Yamauchi, and M. Doi, "A real-time vision-based interface using motion processor and applications to robotics," in Systems and Computers in Japan, vol. 34, pp. 10-19, 2003.

[11] S. Waldherr, R. Romero, and S. Thrun, "A gesture based interface for human-robot interaction," in Autonomous Robots, vol. 9, no.2, pp. 151-173, Springer, 2000.

[12] J. Aleotti, A. Skoglund and T. Duckett, "Position teaching of a robot arm by demonstration with a wearable input device," in International Conference on Intelligent Manipulation and Grasping (IMG04), Genoa, Italy, July 1-2, 2004.

[13] S. Calinon, and A. Billard, "Active teaching in robot programming by demonstration," in 16th IEEE International Symposium on Robot and Human interactive Communication, RO-MAN 2007, pp. 702707, Jeju Island, Korea, 2008

[14] S. Perrin, A. Cassinelli, and M. Ishikawa, "Gesture recognition using laser-based tracking system," in Sixth IEEE International Conference on Automatic Face and Gesture Recognition, pp. 541-546, 2004.

[15] X. Teng, B. Wu, W. Yu, and C. Liu, "A hand gesture recognition system based on local linear embedding", Journal of Visual Languages \& Computing, Vol. 16, pp. 442-454, 2005

[16] J. Chae, H. Kulah, and K. Najafi, "A Hybrid Silicon-OnGlass (SOG) Lateral Micro-Accelerometer With CMOS Readout Circuitry," Technical Digest, IEEE 2002 Int Conference on Micro Electro Mechanical Systems (MEMS 2002), Las Vegas, January 2002

[17] J. Chae, H. Kulah, and K. Najafi, "An In-Plane HighSensitivity, Low-Noise Micro-g Silicon Accelerometer with CMOS Readout Circuitry," IEEE/ASME Journal of Microelectromechanical Systems (JMEMS), vol. 13, no. 4, pp.628-635, August, 2004

[18] J. Chae, H. Kulah, and K. Najafi, "A CMOS-compatible high aspect ratio silicon-on-glass in-plane microaccelerometer," J. Micromechanics and Microengineering, 15 , No. 2, pp. 336-345

[19] Bao, L. and Intille, S. 2004. Activity Recognition from User-Annotated Acceleration Data. Lecture Notes Computer Science 3001, 1-17.

[20] Datasheets of HT-12D, HT-12E, PIC 16F877A, ADXL $335,16 * 2 \mathrm{LCD}$ 